\title{
COMPOSITION OPERATORS ON SOME HOLOMORPHIC BANACH FUNCTION SPACES
}

\author{
A. EL-SAYED AHMED and M. A. BAKHIT
}

\begin{abstract}
In this paper, we study composition operators on some Möbius invariant Banach function spaces like Bloch and $F(p, q, s)$ spaces. We give a Carleson measure characterization on $F(p, q, s)$ spaces, then we use this Carleson measure characterization of the compact compositions on $F(p, q, s)$ spaces to show that every compact composition operator on $F(p, q, s)$ spaces is compact on a Bloch space. Also, we give conditions to clarify when the converse holds.
\end{abstract}

\section{Introduction}

Let $\phi$ be an analytic self-map of the unit disk $\Delta=\{z:|z|<1\}$ in the complex plane $\mathrm{C}$ and let $d A(z)$ be the Euclidean area element on $\Delta$. Associated with $\phi$, the composition operator $C_{\phi}$ is defined by

$$
C_{\phi} f=f \circ \phi,
$$

for $f$ analytic on $\Delta$. It maps analytic functions $f$ to analytic functions. The problem of boundedness and compactness of $C_{\phi}$ has been studied in many function spaces. The first setting was in the Hardy space $H^{2}$, the space of functions analytic on $\Delta$ (see [21]). Madigan and Matheson (see [15]) gave a characterization of the compact composition operators on the Bloch space $\mathscr{B}$. Tjani (see [27]) gave a Carleson measure characterization of compact operators $C_{\phi}$ on Besov spaces $B_{p}(1<p<\infty)$. Bourdon, Cima and Matheson in [7] and Smith in [22] investigated the same problem on BMOA. Li and Wulan in [11] gave a characterization of compact operators $C_{\phi}$ on $Q_{K}$ and $F(p, q, s)$ spaces. In this paper we study compact composition operators on the spaces $F(p, q, s)$, we will define and discuss properties of these spaces, then we give a Carleson measure characterization of the compact composition operator $C_{\phi}$ on $F(p, q, s)$ spaces, see Section 2.

In Section 3, we give another characterization of the compact composition operator on $F(p, q, s)$ spaces.

Received July 17, 2007. 
For $a \in \Delta$ the Möbius transformations $\varphi_{a}(z)$ is defined by

$$
\varphi_{a}(z)=\frac{a-z}{1-\bar{a} z}, \quad \text { for } \quad z \in \Delta .
$$

The following identity is easily verified:

$$
1-\left|\varphi_{a}(z)\right|^{2}=\frac{\left(1-|a|^{2}\right)\left(1-|z|^{2}\right)}{|1-\bar{a} z|^{2}}=\left(1-|z|^{2}\right)\left|\varphi_{a}^{\prime}(z)\right| .
$$

Note that $\varphi_{a}\left(\varphi_{a}(z)\right)=z$ and thus $\varphi_{a}^{-1}(z)=\varphi_{a}(z)$. For $a, z \in \Delta$ and $0<r<1$, the pseudo-hyperbolic disc $\Delta(a, r)$ is defined by $\Delta(a, r)=\left\{z \in \Delta:\left|\varphi_{a}(z)\right|<\right.$ $r\}$. Denote by

$$
g(z, a)=\log \left|\frac{1-\bar{a} z}{z-a}\right|=\log \frac{1}{\left|\varphi_{a}(z)\right|}
$$

the Green function of $\Delta$ with logarithmic singularity at $a \in \Delta$.

Definition 1.1 ([30]). Let $f$ be an analytic function on $\Delta$ and let $0<$ $\alpha<\infty$. If

$$
\|f\|_{\mathscr{B}^{\alpha}}=\sup _{z \in \Delta}\left(1-|z|^{2}\right)^{\alpha}\left|f^{\prime}(z)\right|<\infty
$$

then $f$ belongs to the $\alpha$-Bloch space $\mathscr{B}^{\alpha}$. The space $\mathscr{B}^{1}$ is called the Bloch space $\mathscr{B}$.

Definition 1.2 ([23], [24]). Let $f$ be an analytic function on $\Delta$ and let $1<p<\infty$. If

$$
\|f\|_{B_{p}}^{p}=\sup _{z \in \Delta} \int_{\Delta}\left|f^{\prime}(z)\right|^{p}\left(1-|z|^{2}\right)^{p-2} d A(z)<\infty,
$$

then $f$ belongs to the Besov space $B_{p}$.

Definition 1.3 ([6], [14], [31]). Let $f$ be an analytic function on $\Delta$ and let $0<p<\infty$. If

$$
\int_{\Delta}|f(z)|^{p} d A(z)<\infty
$$

then $f$ belongs to the Bergman space $L_{a}^{p}$.

Definition 1.4 ([21]). Let $f$ be an analytic function on $\Delta$ and let $0<$ $p<\infty$. If

$$
\|f\|_{p}^{p}=\sup _{0<r<1} \frac{1}{2 \pi} \int_{0}^{2 \pi}\left|f\left(r e^{i \theta}\right)\right|^{p} d \theta<\infty,
$$


then $f$ belongs to the Hardy space $H^{p}$. If $\|f\|_{\infty}=\sup _{z \in \Delta}|f(z)|<\infty$, then $f$ belongs to the Hardy space $H^{\infty}$. Moreover, $f \in H^{2}$ if and only if

$$
\sup _{z \in \Delta} \int_{\Delta}\left|f^{\prime}(z)\right|^{2}\left(1-|z|^{2}\right) d A(z)<\infty .
$$

Definition 1.5 ([14]). Let $f(z)=\sum_{k=0}^{\infty} a_{k} z^{k}$ be an analytic function on $\Delta$ and let $-1<q<\infty$. If

$$
\|f\|_{D_{q}}^{2}=\sum_{n=1}^{\infty} n^{1-q}\left|a_{n}\right|^{2}<\infty,
$$

then $f$ belongs to the Dirichlet space $D_{q}$. It is easy to see that $f \in D_{q}$ if and only if

$$
\sup _{z \in \Delta} \int_{\Delta}\left|f^{\prime}(z)\right|^{2}\left(1-|z|^{2}\right)^{q} d A(z)<\infty .
$$

In [29] Zhao gave the following definition:

Definition 1.6. Let $f$ be an analytic function on $\Delta$ and let $0<p<\infty$, $-2<q<\infty$ and $0<s<\infty$. If

$$
\|f\|_{F(p, q, s)}^{p}=\sup _{a \in \Delta} \int_{\Delta}\left|f^{\prime}(z)\right|^{p}\left(1-|z|^{2}\right)^{q} g^{s}(z, a) d A(z)<\infty,
$$

then $f \in F(p, q, s)$. Moreover, if

$$
\lim _{|a| \rightarrow 1} \int_{\Delta}\left|f^{\prime}(z)\right|^{p}\left(1-|z|^{2}\right)^{q} g^{s}(z, a) d A(z)=0,
$$

then $f \in F_{0}(p, q, s)$.

The spaces $F(p, q, s)$ were intensively studied by Zhao in [29] and Rättyä in [18]. It is known from ([29], Theorem 2.10) that, for $p \geq 1$, the spaces $F(p, q, s)$ are Banach spaces under the norm

$$
\|f\|=\|f\|_{F(p, q, s)}+|f(0)| .
$$

Moreover, it is known that in Definition 1.6 the Green function $g(z, a)$ can be replaced by the weight function $1-\left|\varphi_{a}(z)\right|^{2}$ and that for $q+s \leq-1$ the spaces $F(p, q, s)$ and $F_{0}(p, q, s)$ both reduce to the space of constant functions (see [29], Theorem 2.4 and Proposition 2.12). It is sometimes convenient to replace the parameter $q$ by $p-2$ and consider the spaces $F(p, p-2, s)$ and $F_{0}(p, p-2, s)$ instead of the spaces $F(p, q, s)$ and $F_{0}(p, q, s)$ (see [18]). If 
$q=p-2$ and $s=0$, we denote $F(p, p-2,0)=F_{0}(p, p-2,0)=\mathbf{B}_{p}$. The Besov-spaces have been studied by many authors, for example in [1], [2], [25], [26], [31] and [32]. If $p=2$ the spaces $F(2,0, s)$ and $F_{0}(2,0, s)$ are denoted by $Q_{s}$ and $Q_{s, 0}$. The spaces $Q_{s}$ and $Q_{s, 0}$ were introduced by Aulaskari et al. (see [3] and [4] respectively).

Zhao in ([29], Proposition 4.3) showed that all $F(p, q, s)$ are $\frac{q+2}{p}$-Möbius invariant spaces. The same author [29] collected the following immediate relations of $F(p, q, s)$ and $F_{0}(p, q, s)$ (see also [13]):

(1) $F(p, q, s)=\mathscr{B}^{(q+2) / p}$ and $F_{0}(p, q, s)=\mathscr{B}_{0}^{(q+2) / p}$, for $s>1$.

(2) $F(2,0, s)=Q_{s}, F_{0}(2,0, s)=Q_{s, 0}$ and then an analytic function $f$ : $\Delta \rightarrow \mathrm{C}$ defined on the unit disk $\Delta$ belongs to the spaces $F(p, q, s)$ if

$$
\|f\|_{F(p, q, s)}^{p}=\sup _{a \in \Delta} \int_{\Delta}\left|f^{\prime}(z)\right|^{p}\left(1-|z|^{2}\right)^{q} g^{s}(z, a) d A(z)<\infty,
$$

where $g(z, a)=\log \frac{1}{\left|\varphi_{a}(z)\right|}$ is the Green function of $\Delta$ with logarithmic singularity at $a \in \Delta$.

(3) $F(2,1,0)=H^{2}$.

(4) $F(p, p, 0)=L_{a}^{p}$, for $1 \leq p<\infty$ (see [31], Theorem 4.2.9).

(5) $F(2, q, 0)=D_{q}$, for $-1<q<\infty$.

(6) $F(p, p-2,0)=B_{p}$, for $1<p<\infty$.

The following theorem is useful for our study (see [30]):

Theorem 1.1. Let $0<\alpha<\infty, 0<r<1,0<p<\infty$ and $1<s<\infty$. Then, for an analytic function $f$ in $\Delta$, the following quantities are equivalent:

$$
\begin{gathered}
\|f\|_{\mathscr{B}^{\alpha}} \\
\sup _{a \in \Delta} \frac{1}{|\Delta(a, r)|^{1-\frac{p \alpha}{2}}} \int_{\Delta(a, r)}\left|f^{\prime}(z)\right|^{p} d A(z), \\
\sup _{a \in \Delta} \int_{\Delta(a, r)}\left|f^{\prime}(z)\right|^{p}\left(1-|z|^{2}\right)^{p \alpha-2} d A(z), \\
\sup _{a \in \Delta} \int_{\Delta(a, r)}\left|f^{\prime}(z)\right|^{p}\left(1-|z|^{2}\right)^{p \alpha-2}\left(1-\left|\varphi_{a}(z)\right|^{2}\right)^{s} d A(z), \\
\sup _{a \in \Delta} \int_{\Delta(a, r)}\left|f^{\prime}(z)\right|^{p}\left(1-|z|^{2}\right)^{p \alpha-2} g^{s}(z, a) d A(z), \\
\sup _{a \in \Delta} \int_{\Delta(a, r)}\left|f^{\prime}(z)\right|^{p}\left(\log \frac{1}{|z|}\right)^{p \alpha}\left|\varphi_{a}^{\prime}(z)\right|^{2} d A(z) .
\end{gathered}
$$


We will need the following lemma:

Lemma 1.1 (see [27]). Let X, Y be two Banach spaces of analytic functions on $\Delta$. Suppose

(i) the point evaluation functionals on $X$ are continuous,

(ii) the closed unit ball of $X$ is a compact subset of $X$ in the topology of uniform convergence on compact sets,

(iii) $T: X \rightarrow Y$ is continuous when $X$ and $Y$ are given the topology of uniform convergence on compact sets.

Then $T$ is a compact operator if and only if given a bounded sequence $\left(f_{n}\right)$ in $X$ such that $f_{n} \rightarrow 0$ uniformly on compact sets, then the sequence $\left(T f_{n}\right)$ converges to zero in the norm of $Y$.

Proof. This Lemma is proved by Tjani in [27].

Recall that a linear operator $T: X \rightarrow Y$ is said to be compact if it takes bounded sets in $X$ to sets in $Y$ which have compact closure. For Banach spaces $X$ and $Y$ of the space of all analytic functions $H(\Delta)$, we say that $T$ is compact from $X$ to $Y$ if and only if for each bounded sequence $\left\{x_{n}\right\}$ in $X$, the sequence $\left\{T x_{n}\right\} \in Y$ contains a subsequence converging to some limit in $Y$.

\section{Carleson measures and compact composition operators}

Shapiro solved the compactness problem for composition operators on Hardy spaces in [21] using the Nevanlinna counting function $N_{\phi}(w)=\sum_{\phi(z)=w}-\log |z|$. Madigan and Matheson characterize compact composition operators in the Bloch space (see [15]). Tjani characterized the compact composition operators on Besov spaces in [27] using the Nevanlinna type counting function for the $p$-Besov space $B_{p}$ is

$$
N_{p}(w, \phi)=\sum_{\phi(z)=w}\left\{\left|\phi^{\prime}(z)\right|\left(1-|z|^{2}\right)\right\}^{p-2} \quad \text { for } \quad w \in \Delta, \quad p>1
$$

In [11] Li and Wulan gave a modification of the Nevanlinna type counting function and they used it to characterize the compact composition operators on $F(p, q, s)$ as follows:

Definition 2.1 (see [11]). The counting function for the $F(p, q, s)$ spaces is

$$
N_{p, q, s, \phi}(w)=\sum_{\phi(z)=w}\left\{\left|\phi^{\prime}(z)\right|^{p-2}\left(1-|z|^{2}\right)^{q} g^{s}(z, a)\right\},
$$


for $w \in \phi(\Delta), 2 \leq p<\infty,-2<q<\infty$ and $0<s<\infty$.

The above counting functions come up in the change of variables formula in the respective spaces as follows: For $f \in F(p, q, s), 2 \leq p<\infty,-2<$ $q<\infty, 0<s<\infty$ and $q+s>-1$,

$$
\begin{aligned}
\left\|C_{\phi} f\right\|_{F(p, q, s)}^{p} & =\sup _{a \in \Delta} \int_{\Delta}\left|(f \circ \phi)^{\prime}(z)\right|^{p}\left(1-|z|^{2}\right)^{q} g^{s}(z, a) d A(z) \\
& =\sup _{a \in \Delta} \int_{\Delta}\left|f^{\prime}(\phi(z))\right|^{p}\left|\phi^{\prime}(z)\right|^{p}\left(1-|z|^{2}\right)^{q} g^{s}(z, a) d A(z) .
\end{aligned}
$$

By making a non-univalent change of variables as in [21], we see that

$$
\left\|C_{\phi} f\right\|_{F(p, q, s)}^{p}=\sup _{a \in \Delta} \int_{\Delta}\left|f^{\prime}(w)\right|^{p} N_{p, q, s, \phi}(w) d A(w) .
$$

Now we consider the restriction of $C_{\phi}$ to $F(p, q, s)$. Then $C_{\phi}$ is a bounded operator if and only if there is a positive constant $K$ such that

$$
\left\|C_{\phi} f\right\|_{F(p, q, s)}^{p} \leq K\|f\|_{F(p, q, s)}^{p}
$$

for all $f \in F(p, q, s)$ or, equivalently by (3),

$$
\sup _{a \in \Delta} \int_{\Delta}\left|f^{\prime}(w)\right|^{p} N_{p, q, s, \phi}(w) d A(w) \leq K\|f\|_{F(p, q, s)}^{p}
$$

for all $f \in F(p, q, s)$.

Now, let $0<h<1,0 \leq \theta<2 \pi$, and let

$$
\begin{aligned}
\Omega(h, \theta) & =\left\{r e^{i t}: 1-h<r<1 \text { and }|t-\theta|<h\right\}, \\
S(h, \theta) & =\left\{r e^{i t}:\left|r e^{i t}-r e^{i \theta}\right|<h\right\} .
\end{aligned}
$$

A positive measure $\mu$ on $\Delta$ is a Carleson measure if there is a constant $A$ with

$$
\mu(S(h, \theta)) \leq A h, \quad \text { where } 0<h<1 \text { and } 0 \leq \theta<2 \pi .
$$

Here, we shall show that the measures which obey a "generalized" Carleson condition, play a role in understanding which analytic functions $\phi$ mapping $\Delta$ into $\Delta$ produce bounded composition operators on certain Möbius invariant spaces $X=(F(p, q, s)$ or $X=\mathscr{B})$.

This leads, as in [1], to the following definition of generalized Carleson type measures. Since we are interested in characterizing the compact composition operators, we will also talk about vanishing Carleson measures. 
Definition 2.2. Let $\mu$ be a positive measure on $\Delta$, and let $X=\mathscr{B}$ or $F(p, q, s)$ for $0<p<\infty,-2<q<\infty$ and $0<s<\infty$. Then $\mu$ is an $(X, p)$-Carleson measure if there is a constant $A>0$ so that

$$
\int_{\Delta}\left|f^{\prime}(w)\right|^{p} d \mu(w) \leq A\|f\|_{X}^{p}
$$

for all $f \in X$, holds.

Definition 2.3. For $1<p<\infty, \mu$ is called a vanishing $p$-Carleson measure if

$$
\lim _{h \rightarrow 0} \sup _{\theta \in[0,2 \pi)} \frac{\mu(S(h, \theta))}{h^{p}}=0 .
$$

In view of (4) above we see that $C_{\phi}$ is a bounded operator on $F(p, q, s)$ if and only if the measure $N_{p, q, s, \phi}(w) d A(w)$ is a $(F(p, q, s), p)$-Carleson measure. Now we give a characterization of compact composition operators on $F(p, q, s)$ spaces in terms of $p$-Carleson measures.

Theorem 2.1. Let $0<p<\infty$ and $1<s<\infty$. The following are equivalent:

(i) $\mu$ is a $(F(p, p-2, s), p)$-Carleson measure,

(ii) there is a constant $A$ such that $\mu(S(h, \theta)) \leq A h^{p}$ for all $h \in(0,1)$ and all $\theta \in[0,2 \pi)$,

(iii) there is a constant $C$ such that

$$
\sup _{a \in \Delta} \int_{\Delta}\left|\varphi_{a}^{\prime}(z)\right|^{p} d \mu(z) \leq C \quad \text { for all } a \in \Delta .
$$

Proof. Suppose (i) holds. Then using Theorem 1.1 and Definition 2.2, we obtain

$$
\int_{\Delta}\left|f^{\prime}(z)\right|^{p} d \mu(z) \leq C \int_{\Delta}\left|f^{\prime}(z)\right|^{p}\left(1-|z|^{2}\right)^{p-2} g^{s}(z, a) d A(z),
$$

for all $f \in F(p, p-2, s)$. In particular this holds for $f(z)=\varphi_{a}(z)=\frac{a-z}{1-\bar{a} z}$. Hence

$$
\begin{aligned}
\sup _{a \in \Delta} \int_{\Delta}\left|\varphi_{a}^{\prime}(z)\right|^{p} d \mu(z) & \leq C \sup _{a \in \Delta} \int_{\Delta}\left|\varphi_{a}^{\prime}(z)\right|^{p}\left(1-|z|^{2}\right)^{p-2} g^{s}(z, a) d A(z) \\
& \leq C\left\|\varphi_{a}\right\|_{F(p, p-2, s)}^{p} \leq C \text { const. }
\end{aligned}
$$


for all $a \in \Delta$. This gives (iii). The equivalence of (ii) and (iii) was given by Arazy, Fisher and Peetre in ([1] Theorem 13). Suppose now that (ii) holds; we shall show that (i) is true, thus completing the implications. For $z=r e^{i \theta}$, let $E_{1}(z)=\left\{w:|w-z|<\frac{1-|z|}{2}\right\} \quad$ and $\quad E_{2}(z)=\{w:|w-z|<1-|z|\}$.

Then

$$
E_{1}(z) \subseteq E_{2}(z) \subseteq S(2(1-|z|), \theta)
$$

Further, if $w \in E_{1}(z)$, then

$$
\frac{1}{2} \leq \frac{1-|w|}{1-|z|} \leq \frac{3}{2}
$$

Let $f \in F(p, q, s)$; because $f$ is analytic we have

$$
f^{\prime}(z)=\frac{4}{\pi(1-|z|)^{2}} \int_{E_{1}(z)} f^{\prime}(w) d A(w) .
$$

Therefore by Jensen's inequality (see [20]),

$$
\left|f^{\prime}(z)\right|^{p} \leq \frac{4}{\pi(1-|z|)^{2}} \int_{E_{1}(z)}\left|f^{\prime}(w)\right|^{p} d A(w) .
$$

Thus,

$$
\begin{aligned}
\int_{\Delta}\left|f^{\prime}(z)\right|^{p} d \mu(z) & \leq \int_{\Delta} \frac{4}{\pi(1-|z|)^{2}}\left(\int_{E_{1}(z)}\left|f^{\prime}(w)\right|^{p} d A(w)\right) d \mu(z) \\
& \leq \frac{4}{\pi} \int_{\Delta}\left(\int_{E_{1}(z)}\left|f^{\prime}(w)\right|^{p}\left(\frac{3}{2(1-|w|)}\right)^{2} d A(w)\right) d \mu(z) \\
& \leq \frac{9}{\pi} \int_{\Delta} \int_{\Delta}\left|f^{\prime}(w)\right|^{p} \chi_{E_{1}(z)}(w)(1-|w|)^{-2} d A(w) d \mu(z) \\
& \leq \frac{9}{\pi} \int_{\Delta}\left|f^{\prime}(w)\right|^{p}(1-|w|)^{-2} \int_{\Delta} \chi_{E_{1}(z)}(w) d \mu(z) d A(w) .
\end{aligned}
$$

However, $\chi_{E_{1}(z)}(w) \leq \chi_{S(2(1-|z|), \theta)}(w), z=|z| e^{i \theta}$, since $w \in E_{1}(z)$ implies that

$$
\left|w-e^{i \theta}\right|<2(1-|w|)
$$

Now applying (ii) we have

$$
\int_{\Delta} \chi_{E_{1}(z)} d \mu(z) \leq \mu(S(2(1-|w|), \theta)) \leq A 2^{p}(1-|w|)^{p} .
$$


Therefore,

$$
\begin{aligned}
\int_{\Delta}\left|f^{\prime}(z)\right|^{p} d \mu(z) & \leq \frac{9}{\pi} A 2^{p} \int_{\Delta}\left|f^{\prime}(w)\right|^{p}(1-|w|)^{p-2} d A(w) \\
& \leq C \int_{\Delta}\left|f^{\prime}(w)\right|^{p}(1-|w|)^{p-2} d A(w)
\end{aligned}
$$

where $C$ is a constant. By Theorem 1.1 the quantities $(\mathrm{C})$ and $(\mathrm{E})$ are equivalent so, for $\alpha=1$, we have

$$
\begin{aligned}
\int_{\Delta}\left|f^{\prime}(z)\right|^{p} d \mu(z) & \leq C \int_{\Delta}\left|f^{\prime}(w)\right|^{p}(1-|w|)^{p-2} g^{s}(z, a) d A(w) \\
& \leq C\|f\|_{F(p, p-2, s)},
\end{aligned}
$$

which is (i). This finishes the proof.

Hence, Theorem 2.1 yields:

THEOREM 2.2. Let $\phi$ be an analytic function on $\Delta, 0<p<\infty$, and $1<s<\infty$. Then $C_{\phi}$ is a bounded operator on $F(p, p-2, s)$ if and only if

$$
\sup _{a \in \Delta}\left\|C_{\phi} \varphi_{a}\right\|_{F(p, p-2, s)}<\infty .
$$

The following proposition comes from ([5], Lemma 2.1):

Proposition 2.1. For $0<p<\infty$, a positive measure $\mu$ on $\Delta$ is a bounded $p$-Carleson measure if and only if

$$
\sup _{a \in \Delta} \int_{\Delta}\left|\varphi_{a}^{\prime}(z)\right|^{p} d \mu(z)<\infty ;
$$

$\mu$ is a compact p-Carleson measure if and only if

$$
\lim _{|a| \rightarrow 1} \int_{\Delta}\left|\varphi_{a}^{\prime}(z)\right|^{p} d \mu(z)=0 .
$$

Arazy, Fisher, and Peetre in [1], Cima and Wogen in [8], Tjani in [27] gave the characterization of the $p$-Carleson measure on Besov spaces.

We will prove the following lemmas on $F(p, q, s)$ spaces:

Lemma 2.1. Let $X=F(p, q, s)$ where $2 \leq p<\infty, 0<q<\infty$, $0<s<\infty$. Then

(i) Every bounded sequence $\left(f_{n}\right) \subset X$ is uniformly bounded on compact sets. 
(ii) For any sequence $\left(f_{n}\right)$ on $X$ such that $\left\|f_{n}\right\|_{X} \rightarrow 0, f_{n}-f_{n}(0) \rightarrow 0$ uniformly on compact sets.

Proof. In [29] it is shown for $\alpha=(q+2) / p$ that,

$$
\|f\|_{\mathscr{B}^{\alpha}} \leq M(p, q, s)\|f\|_{F(p, q, s)},
$$

where $M(p, q, s)$ is a constant depending on $p, q$ and $s$. If $z \in \Delta(0, r)$, $0<r<1$, then we have

$$
\begin{aligned}
\left|f_{n}-f_{n}(0)\right|=\left|\int_{0}^{1} f_{n}^{\prime}(z t) z d t\right| & \leq\left\|f_{n}\right\|_{\mathscr{B}^{\alpha}} \int_{0}^{1} \frac{|z| d t}{\left(1-|z|^{2} t^{2}\right)^{\alpha}} \\
& \leq\left\|f_{n}\right\|_{\mathscr{B}^{\alpha}} \frac{1}{\left(1-|r|^{2}\right)^{\alpha}} \\
& \leq\left\|f_{n}\right\|_{F(p, q, s)} M(p, q, s) \frac{1}{\left(1-|r|^{2}\right)^{\alpha}} .
\end{aligned}
$$

Hence the result follows.

Lemma 2.2. Let $X, Y=F(p, q, s)(2 \leq p<\infty, 0<q<\infty, 0<$ $s<\infty)$ or $\mathscr{B}$. Then $C_{\phi}: X \rightarrow Y$ is a compact operator if and only if for any bounded sequence $\left(f_{n}\right) \subset X$ with $f_{n} \rightarrow 0$ uniformly on compact sets as $n \rightarrow \infty,\left\|C_{\phi} f_{n}\right\|_{Y} \rightarrow 0$ as $n \rightarrow \infty$.

Proof. We will show that (i), (ii), and (iii) of Lemma 1.1 hold for our spaces. By Lemma 2.1 it is easy to see that (i) and (iii) hold. To show that (ii) holds, let $\left(f_{n}\right)$ be a sequence in the closed unit ball of $X$. Then by Lemma 2.1, $\left(f_{n}\right)$ is uniformly bounded on compact sets. Therefore, by Montel's theorem (see [9]), there is a subsequence $\left(f_{n_{k}}\right), n_{1}<n_{2}<\cdots$, such that $f_{n_{k}} \rightarrow h$ uniformly on compact sets, for some $h \in H(\Delta)$. Thus we only need to show that $h \in X$.

(a) If $X=F(p, q, s),(2 \leq p<\infty,-2<q<\infty, 0<s<\infty)$, then

$$
\begin{aligned}
& \int_{\Delta}\left|h^{\prime}(z)\right|^{p}\left(1-|z|^{2}\right)^{q} g^{s}(z, a) d A(z) \\
& \quad=\int_{\Delta} \lim _{k \rightarrow \infty}\left|f_{n_{k}}^{\prime}(z)\right|^{p}\left(1-|z|^{2}\right)^{q} g^{s}(z, a) d A(z) \\
& \quad \leq \liminf _{k \rightarrow \infty} \int_{\Delta}\left|f_{n_{k}}^{\prime}(z)\right|^{p}\left(1-|z|^{2}\right)^{q} g^{s}(z, a) d A(z), \\
& \quad=\liminf _{k \rightarrow \infty}\left\|f_{n_{k}}\right\|_{F(p, q, s)}^{p}<\infty,
\end{aligned}
$$

where we used Fatou's theorem and our hypothesis. 
(b) If $X=\mathscr{B}$ as in [27] we have that

$$
\left|h^{\prime}(z)\right|\left(1-|z|^{2}\right)=\lim _{k \rightarrow \infty}\left|f_{n_{k}}^{\prime}(z)\right|\left(1-|z|^{2}\right) \leq \lim _{k \rightarrow \infty}\left\|f_{n_{k}}^{\prime}\right\|_{\mathscr{B}}<\infty,
$$

this by our hypothesis. Therefore, Lemma 2.1 yields that $C_{\phi}: X \rightarrow Y$ is a compact operator if and only if for any bounded sequence $\left(f_{n}\right) \subset X$, with $f_{n} \rightarrow 0$ uniformly on compact sets as $n \rightarrow \infty,\left|f_{n}(\phi(0))\right|+\left\|C_{\phi} f_{n}\right\|_{Y} \rightarrow 0$, as $n \rightarrow \infty$, which is clearly equivalent to the statement of this lemma. This completes the proof of the lemma.

We prove a similar theorem for compact composition operators on $F(p, q, s)$ spaces.

Theorem 2.3. Let $2 \leq p<p^{*}<\infty,-2<q<\infty, 0<s<\infty$. Then the following are equivalent:

(i) $C_{\phi}: F(p, q, s) \rightarrow F\left(p^{*}, q, s\right)$ is a compact operator.

(ii) $N_{p^{*}, q, s, \phi}(w) d A(w)$ is a vanishing $p$-Carleson measure.

(iii) $\left\|C_{\phi} \varphi_{a}\right\|_{F\left(p^{*}, q, s\right)} \rightarrow 0$ as $|a| \rightarrow 1$.

Proof. By (3)

$$
\left\|C_{\phi} \varphi_{a}\right\|_{F\left(p^{*}, q, s\right)}^{p^{*}}=\sup _{a \in \Delta} \int_{\Delta}\left|\varphi_{a}^{\prime}(w)\right|^{p^{*}} N_{p^{*}, q, s, \phi}(w) d A(w)
$$

Thus Proposition 2.1 yields (ii) $\Leftrightarrow$ (iii). Next we show that (i) $\Rightarrow$ (iii).

We assume that $C_{\phi}: F(p, q, s) \rightarrow F\left(p^{*}, q, s\right)$ is a compact operator. Note that $\left\{\varphi_{a}: a \in \Delta\right\}$ is a bounded set in $F(p, q, s)$. Since

$$
\left\|\varphi_{a}\right\|_{F(p, q, s)}=\left\|z \circ \varphi_{a}\right\|_{F(p, q, s)},
$$

the norm of $\varphi_{a}$ in $F(p, q, s)$ is

$$
\left|\varphi_{a}(0)\right|+\left\|\varphi_{a}\right\|_{F(p, q, s)}<1+\left\|\varphi_{a}\right\|_{F(p, q, s)}<\infty .
$$

Also $\left(\varphi_{a}-a\right) \rightarrow 0$ as $|a| \rightarrow 1$, uniformly on compact sets, since

$$
\left|\varphi_{a}-a\right|=|z| \frac{1-|a|^{2}}{|1-\bar{a} z|}, \quad \text { where } \quad|z|=r<1 .
$$

Hence by Lemma 2.2, we obtain that

$$
\left\|C_{\phi}\left(\varphi_{a}-a\right)\right\|_{F\left(p^{*}, q, s\right)} \rightarrow 0, \quad \text { as } \quad|a| \rightarrow 1 .
$$


Finally, let us show that (ii) $\Rightarrow$ (i). Let $\left(f_{n}\right)$ be a bounded sequence in $F(p, q, s)$ that converges to 0 uniformly on compact sets. Then the mean value property for the analytic function $f_{n}^{\prime}$ yields that

$$
f_{n}^{\prime}(w)=\frac{4}{\pi(1-|w|)^{2}} \int_{|w-z|<\frac{1-|w|}{2}}\left|f_{n}^{\prime}(z)\right| d A(z) .
$$

Therefore by Jensen's inequality (see [20], Theorem 3.3) and (5), where

$$
E_{1}(w)=\left\{z:|w-z|<\frac{1-|w|}{2}\right\}
$$

$$
\left|f_{n}^{\prime}(w)\right|^{p^{*}} \leq \frac{4}{\pi(1-|w|)^{2}} \int_{E_{1}(w)}\left|f_{n}^{\prime}(z)\right|^{p^{*}} d A(z) .
$$

Then by (6) and Fubini's Theorem (see [20], Theorem 8.8),

$$
\begin{aligned}
& \left\|C_{\phi} f_{n}\right\|_{F\left(p^{*}, q, s\right)}^{p^{*}} \\
& \quad=\sup _{a \in \Delta} \int_{\Delta}\left|f_{n}^{\prime}(w)\right|^{p^{*}} N_{p^{*}, q, s, \phi}(w) d A(w) \\
& \quad \leq \sup _{a \in \Delta} \int_{\Delta} \frac{4}{\pi(1-|w|)^{2}}\left(\int_{E_{1}(w)}\left|f_{n}^{\prime}(z)\right|^{p^{*}} d A(z)\right) N_{p^{*}, q, s, \phi}(w) d A(w) .
\end{aligned}
$$

Then,

$$
\begin{aligned}
& \text { (7) }\left\|C_{\phi} f_{n}\right\|_{F\left(p^{*}, q, s\right)}^{p^{*}} \\
& \leq \frac{4}{\pi} \sup _{a \in \Delta} \int_{\Delta}\left|f_{n}^{\prime}(z)\right|^{p^{*}}\left(\int_{\Delta} \frac{1}{(1-|w|)^{2}} \chi_{E_{1}(w)}(z) N_{p^{*}, q, s, \phi}(w) d A(w)\right) d A(z),
\end{aligned}
$$

Note that if $|w-z|<\frac{1-|w|}{2}$, then $w \in S(2(1-|z|), \theta)$, where $z=|z| e^{i \theta}$, since

$$
\left|w-e^{i \theta}\right| \leq|z-w|+\left|e^{i \theta}-z\right|<\frac{1-|w|}{2}+\left|\frac{z}{|z|}-z\right|<2(1-|z|) .
$$

Moreover, if $|w-z|<\frac{1-|w|}{2}$, then $\frac{1}{(1-|w|)^{2}}<$ const. $\frac{1}{(1-|z|)^{2}}$. Therefore, (5) 
yields

$$
\begin{aligned}
& \left\|C_{\phi} f_{n}\right\|_{F\left(p^{*}, q, s\right)}^{p^{*}} \\
& \leq \text { const. } \sup _{a \in \Delta} \int_{\Delta} \frac{\left|f_{n}^{\prime}(z)\right|^{p^{*}}}{(1-|z|)^{2}}\left(\int_{S(2(1-|z|), \theta)} N_{p^{*}, q, s, \phi}(w) d A(w)\right) d A(z) \\
& =\text { const. } \sup _{a \in \Delta}\left(\int_{|z|>1-\frac{\delta}{2}}\right. \\
& \left.\quad+\int_{|z| \leq 1-\frac{\delta}{2}} \frac{\left|f_{n}^{\prime}(z)\right|^{p^{*}}}{(1-|z|)^{2}}\left(\int_{S(2(1-|z|), \theta)} N_{p^{*}, q, s, \phi}(w) d A(w)\right) d A(z)\right) \\
& =\text { const. } \sup _{a \in \Delta}(I+I I),
\end{aligned}
$$

for any $0<\delta<1$. Fix $\varepsilon>0$ and let $\delta>0$ be such that for any $\theta \in[0,2 \pi]$ and any $h<\delta$,

$$
\sup _{a \in \Delta} \int_{S(h, \theta)} N_{p^{*}, q, s, \phi}(w) d A(w) \leq \varepsilon h^{p^{*}}
$$

By (8) we have

$$
\begin{aligned}
I & \leq \varepsilon 2^{p^{*}} \int_{|z|>1-\frac{\delta}{2}} \frac{\left|f_{n}^{\prime}(z)\right|^{p^{*}}}{(1-|z|)^{2}}(1-|z|)^{p^{*}} d A(z) \\
& \leq \varepsilon 2^{p^{*}} \int_{|z|>1-\frac{\delta}{2}}\left|f_{n}^{\prime}(z)\right|^{p^{*}}(1-|z|)^{p^{*}-2} d A(z) \\
& \leq \varepsilon \text { const. }\left\|f_{n}\right\|_{B_{p^{*}}}^{p^{*}}<\varepsilon \text { const. },
\end{aligned}
$$

and

$$
\begin{aligned}
I I & \leq \text { const. } \sup _{a \in \Delta} \int_{|z| \leq 1-\frac{\delta}{2}} \frac{\left|f_{n}^{\prime}(z)\right|^{p^{*}}}{(1-|z|)^{2}}\left(\int_{S(2(1-|z|), \theta)} N_{p^{*}, q, s, \phi}(w) d A(w)\right) d A(z) \\
& =\text { const. } \sup _{a \in \Delta}\left(\int_{\Delta} N_{p^{*}, q, s, \phi}(w) d A(w)\right) \int_{|z| \leq 1-\frac{\delta}{2}}\left|f_{n}^{\prime}(z)\right|^{p^{*}} d A(z)<\text { const. }
\end{aligned}
$$

for $n$ large enough, since $f_{n}^{\prime} \rightarrow 0$ uniformly on compact sets. We obtain that

$$
\left\|C_{\phi} f_{n}\right\|_{F\left(p^{*}, q, s\right)}^{p^{*}}<\text { const. } \sup _{a \in \Delta}(I+I I)<\varepsilon \text { const. }
$$


for $n$ large enough. Therefore, $\left\|C_{\phi} f_{n}\right\|_{F\left(p^{*}, q, s\right)}^{p^{*}} \rightarrow 0$, as $n \rightarrow \infty$ and Lemma 3.2 yields that $C_{\phi}: F(p, q, s) \rightarrow F\left(p^{*}, q, s\right)$ is a compact operator. This finishes the proof of the theorem.

\section{3. $F(p, q, s)$ compactness of $C_{\phi}$ versus Bloch compactness of $C_{\phi}$}

Several authors have obtained some characterizations of composition operators (see e.g. [7], [10], [11], [12], [16], [19], [21], [28] and others). Recall the characterization of compact composition operators on the Bloch space that Madigan and Matheson obtained in [15], Theorem 2.

Theorem 3.1 (see [15]). Let $\phi$ be a analytic function on $\Delta$. Then $C_{\phi}$ is a compact operator on Bloch space if and only if

$$
\lim _{|z| \rightarrow 1} \frac{\left|\phi^{\prime}(z)\right|\left(1-|z|^{2}\right)}{1-|\phi(z)|^{2}}=0
$$

Tjani gave another characterization of compact composition operators on Besov spaces and Bloch spaces in [27].

TheOREM 3.2 (see [27]). Let $\phi$ be an analytic self-map of $\Delta$. Let $X=B_{p}$, $1<p<\infty$, or $\mathscr{B}$. Then $C_{\phi}: X \rightarrow \mathscr{B}$ is a compact operator if and only if

$$
\left\|C_{\phi} \varphi_{a}\right\|_{\mathscr{B}} \rightarrow 0 \quad \text { as } \quad|a| \rightarrow 1 .
$$

Now we give another characterization of compact composition operators on the $F(p, q, s)$ spaces and the Bloch space.

Theorem 3.3. Let $2 \leq p<\infty,-2<q<\infty$ and $0<s<\infty$ and let $X=F(p, q, s)$ or $\mathscr{B}$. Let $\phi$ be an analytic self-map of $\Delta$. Then $C_{\phi}: X \rightarrow \mathscr{B}$ is a compact operator if and only if

$$
\left\|C_{\phi} \varphi_{a}\right\|_{\mathscr{B}} \rightarrow 0 \quad \text { as } \quad|a| \rightarrow 1 .
$$

Proof. First, we suppose that $C_{\phi}: X \rightarrow \mathscr{B}$ is a compact operator. Then $\left\{\varphi_{a}(z): a \in \Delta\right\}$ is a bounded set in $F(p, q, s)$ or $\mathscr{B}$, and $\varphi_{a}-a \rightarrow 0$ uniformly on compact sets as $|a| \rightarrow 1$. Thus by Lemma 2.2,

$$
\lim _{|a| \rightarrow 1}\left\|C_{\phi} \varphi_{a}\right\|_{\mathscr{B}}=0 .
$$

Conversely, as in [27], we suppose that

$$
\lim _{|a| \rightarrow 1}\left\|C_{\phi} \varphi_{a}\right\|_{\mathscr{B}}=0 \quad \text { as } \quad|a| \rightarrow 1 .
$$


Let $\left(f_{n}\right)$ be a bounded sequence in $F(p, q, s)$ or $\mathscr{B}$ such that $f_{n} \rightarrow 0$ uniformly on compact sets, as $n \rightarrow \infty$. We will show that

$$
\lim _{n \rightarrow 0}\left\|C_{\phi} f_{n}\right\|_{\mathscr{B}}=0 .
$$

Let $\varepsilon>0$ be given and fix $0<\delta<1$ such that if $|a|>\delta$, then $\left\|C_{\phi} \varphi_{a}\right\|_{\mathscr{B}}<\varepsilon$. Hence for any $z_{0} \in \Delta$ such that $\left|\phi\left(z_{0}\right)\right|>\delta,\left\|C_{\phi} \varphi_{\phi\left(z_{0}\right)}\right\|_{\mathscr{B}}<\varepsilon$. In particular,

$$
\left|\varphi_{\phi\left(z_{0}\right)}^{\prime}\left(\phi\left(z_{0}\right)\right)\right|\left|\phi^{\prime}\left(z_{0}\right)\right|\left(1-\left|z_{0}\right|^{2}\right)<\varepsilon,
$$

that is,

$$
\frac{\left|\phi^{\prime}\left(z_{0}\right)\right|}{1-\left|\phi\left(z_{0}\right)\right|^{2}}\left(1-\left|z_{0}\right|^{2}\right)<\varepsilon
$$

Then (9) yields that for any $n \in \mathrm{N}$ and $z_{0} \in \Delta$ such that $\left|\phi\left(z_{0}\right)\right|>\delta$,

$$
\begin{aligned}
\left|\left(f_{n} \circ \phi\right)^{\prime}\left(z_{0}\right)\right|\left(1-\left|z_{0}\right|^{2}\right) & =\left|f_{n}^{\prime}\left(\phi\left(z_{0}\right)\right)\right|\left|\phi^{\prime}\left(z_{0}\right)\right|\left(1-\left|z_{0}\right|^{2}\right) \\
& <\varepsilon\left|f_{n}^{\prime}\left(\phi\left(z_{0}\right)\right)\right|\left(1-\left|\phi\left(z_{0}\right)\right|^{2}\right) \\
& \leq \varepsilon\left\|f_{n}\right\|_{\mathscr{B}} \leq \varepsilon \text { const. }
\end{aligned}
$$

we obtain that

$$
\left|\left(f_{n} \circ \phi\right)^{\prime}\left(z_{0}\right)\right|\left(1-\left|z_{0}\right|^{2}\right) \leq \varepsilon \text { const. }
$$

Since the set $A=\{w:|w| \leq \delta\}$ is a compact subset of $\Delta$ and $f_{n}^{\prime} \rightarrow 0$ uniformly on compact sets,

$$
\sup _{w \in A}\left|f_{n}^{\prime}(w)\right| \rightarrow 0 \quad \text { as } \quad n \rightarrow \infty .
$$

Therefore we may choose $n_{0}$ large enough so that $\left|f_{n}^{\prime}(\phi(z))\right|<\varepsilon$, for any $n>n_{0}$ and any $z \in \Delta$ such that $|\phi(z)| \leq \delta$. Then, for all such $z$,

$$
\begin{aligned}
\left|\left(f_{n} \circ \phi\right)^{\prime}(z)\right|\left(1-|z|^{2}\right) & =\mid\left(f_{n}^{\prime}(\phi(z))|| \phi^{\prime}(z) \mid\left(1-|z|^{2}\right)\right. \\
& <\varepsilon\left|\phi^{\prime}(z)\right|\left(1-|z|^{2}\right) \\
& \leq \varepsilon\|\phi\|_{\mathscr{B}}<\varepsilon \text { const. }
\end{aligned}
$$

then, where $n \geq n_{0}$

$$
\left|\left(f_{n} \circ \phi\right)^{\prime}(z)\right|\left(1-|z|^{2}\right)<\varepsilon \text { const. }
$$

Thus (10) and (11) yield

$$
\left\|f_{n} \circ \phi\right\|_{\mathscr{B}}=\left\|C_{\phi} f_{n}\right\|_{\mathscr{B}}<\varepsilon \text { const. } \quad \text { for } \quad n \geq n_{0} .
$$


Thus (12) yield that $\left\|C_{\phi} f_{n}\right\|_{\mathscr{B}} \rightarrow 0$ as $n \rightarrow \infty$. Hence by Lemma 2.2, $C_{\phi}$ : $F(p, q, s) \rightarrow \mathscr{B}$ is a compact operator.

Remark 3.1. Theorem 2.1 and Theorem 3.3 show that the compactness of $C_{\phi}$ on $F(p, q, s)$ spaces, and its upper limit the Bloch space, depend on the behavior of the norm of the image under $C_{\phi}$ of the conformal automorphisms $\varphi_{a}$, for $|a| \rightarrow 1$. An immediate corollary of the two theorems is that if $C_{\phi}$ is compact on $F(p, q, s)$ spaces, then it is compact on some $F(p, q, s)$ space with a larger index, and it is compact on the Bloch space. The converse holds if we suppose that $C_{\phi}$ is bounded on some $F(p, q, s)$ space with a smaller index (see Proposition 3.3).

REMARK 3.2. The proof of Theorem 3.3 yields that

$$
\lim _{|a| \rightarrow 1}\left\|C_{\phi} \varphi_{a}\right\|_{\mathscr{B}}=0 \quad \text { if and only if } \quad \lim _{|\phi(z)| \rightarrow 1} \frac{\left|\phi^{\prime}(z)\right|\left(1-|z|^{2}\right)}{1-|\phi(z)|^{2}}=0 .
$$

An immediate consequence of Theorem 2.1 and Theorem 3.3 is the following proposition:

Proposition 3.1. Let $2 \leq p<p^{*}<\infty,-2<q<\infty$ and $0<s<\infty$. Then $C_{\phi}: F(p, q, s) \rightarrow F\left(p^{*}, q, s\right)$ is a compact operator, and so is $C_{\phi}$ : $\mathscr{B} \rightarrow \mathscr{B}$.

Recently Rättyä (see Proposition 3 in [19]) gave a characterization of composition operators acting from the weighted Bergman or Dirichlet space into the BMOA space, the space of analytic functions of bounded mean oscillation. For $F(p, q, s)$ spaces we give the following result:

Proposition 3.2. Let $2 \leq p<p^{*}<\infty,-2<q<\infty$ and $0<s<\infty$. If

$$
\lim _{|w| \rightarrow 1} \sup _{a \in \Delta} \frac{N_{p^{*}, q, s, \phi}(w)}{\left(1-|w|^{2}\right)^{q} g^{s}\left(\phi^{-1}(w), a\right)}=0,
$$

then $C_{\phi}: F(p, q, s) \rightarrow F\left(p^{*}, q, s\right)$ is a compact operator.

Proof. Let $\left(f_{n}\right)$ be a bounded sequence in $F(p, q, s)$ such that $f_{n} \rightarrow 0$ uniformly on compact sets as $n \rightarrow \infty$. Let $\varepsilon>0$ be given and fix $\delta>0$ such that if $1-\delta<|w|<1$, then

$$
N_{p^{*}, q, s, \phi}(w)<\varepsilon\left(1-|w|^{2}\right)^{q} g^{s}\left(\phi^{-1}(w), a\right) .
$$


By (3) we have

$$
\begin{aligned}
\left\|C_{\phi} f_{n}\right\|_{F\left(p^{*}, q, s\right)}^{p^{*}} & =\sup _{a \in \Delta} \int_{\Delta}\left|f_{n}^{\prime}(w)\right|^{p^{*}} N_{p^{*}, q, s, \phi}(w) d A(w) \\
& =\sup _{a \in \Delta} \int_{1-\delta<|w|<1}+\int_{|w| \leq 1-\delta}\left|f_{n}^{\prime}(w)\right|^{p *} N_{p^{*}, q, s, \phi}(w) d A(w),
\end{aligned}
$$

which implies that,

$$
\left\|C_{\phi} f_{n}\right\|_{F\left(p^{*}, q, s\right)}^{p^{*}}=\sup _{a \in \Delta}(I+I I) .
$$

As in ([29] page 33) we determine $b \in \mathrm{C}$ such that $\phi^{-1}(w)=e^{i \theta} \varphi_{b}(w)$. Then it is easy to check that $\varphi_{a}\left(\phi^{-1}(w)\right)=e^{i \gamma} \varphi_{\tilde{a}}(w)$, where $e^{i \gamma}=\frac{a \bar{b}-e^{i \theta}}{1-\bar{a} b e^{i \theta}}$ and $\tilde{a}=\varphi_{b}\left(a e^{-i \theta}\right)$. So, $g\left(\phi^{-1}(w), a\right)=g(w, \tilde{a})$, hence

$$
\begin{aligned}
\sup _{a \in \Delta} \int_{\Delta}\left|f_{n}^{\prime}(w)\right|^{p^{*}}(1- & \left.|w|^{2}\right)^{q} g^{s}\left(\phi^{-1}(w), a\right) d A(w) \\
& =\sup _{\tilde{a} \in \Delta} \int_{\Delta}\left|f_{n}^{\prime}(w)\right|^{p^{*}}\left(1-|w|^{2}\right)^{q} g^{s}(w, \tilde{a}) d A(w) .
\end{aligned}
$$

By (14) and (15), we obtain that

$$
\begin{aligned}
I & \leq \varepsilon \sup _{a \in \Delta} \int_{1-\delta<|w|<1}\left|f_{n}^{\prime}(w)\right|^{p^{*}} N_{p^{*}, q, s, \phi}(w) d A(w) \\
& <\varepsilon \sup _{a \in \Delta} \int_{1-\delta<|w|<1}\left|f_{n}^{\prime}(w)\right|^{p^{*}}\left(1-|w|^{2}\right)^{q} g^{s}\left(\phi^{-1}(w), a\right) d A(w) \\
& =\varepsilon \sup _{\tilde{a} \in \Delta} \int_{1-\delta<|w|<1}\left|f_{n}^{\prime}(w)\right|^{p^{*}}\left(1-|w|^{2}\right)^{q} g^{s}(\phi(w), \tilde{a}) d A(w) .
\end{aligned}
$$

Now $f_{n}$ is bounded in $F\left(p^{*}, q, s\right)$, and then

$$
I<\varepsilon\left\|f_{n}\right\|_{F\left(p^{*}, q, s\right)}^{p *}<\varepsilon \text { const. }
$$

Since $\left|f_{n}^{\prime}\right|^{p^{*}} \rightarrow 0$ uniformly on $\{w \in \Delta:|w|<1-\delta\}$, we can find a positive integer $n_{0}$ such that

$$
I I \leq \varepsilon \sup _{a \in \Delta} \int_{|w| \leq 1-\delta} N_{p^{*}, q, s, \phi}(w) d A(w)<\varepsilon \text { const. }
$$

for $n \geq n_{0}$, since

$$
\sup _{a \in \Delta} \int_{|w| \leq 1-\delta} N_{p^{*}, q, s, \phi}(w) d A(w) \leq\|\phi\|_{F\left(p^{*}, q, s\right)}<\infty .
$$


By (14), (16) and (17)

$$
\left\|C_{\phi} f_{n}\right\|_{F\left(p^{*}, q, s\right)}^{p^{*}}<\varepsilon \text { const. } \quad \text { for } n \geq n_{0} .
$$

Therefore,

$$
\left\|C_{\phi} f_{n}\right\|_{F\left(p^{*}, q, s\right)}^{p^{*}} \rightarrow 0, \quad \text { as } \quad n \rightarrow \infty .
$$

Hence Lemma 2.2 yields that $C_{\phi}: F(p, q, s) \rightarrow F\left(p^{*}, q, s\right)$ is a compact operator. This finishes the proof of the proposition.

THEOREM 3.4. Let $\phi$ be a univalent analytic self-map of $\Delta$. Then for $2 \leq$ $p<\infty, 0<q<\infty$ and $0<s<\infty, C_{\phi}: F(p, q, s) \rightarrow F(p, q, s)$ is a compact operator if and only if $C_{\phi}: \mathscr{B} \rightarrow \mathscr{B}$ is a compact operator with $\lim _{|\phi(z)| \rightarrow 1}\left|\phi^{\prime}(z)\right|=K$, where $K$ is a constant.

Proof. First we suppose that $C_{\phi}$ is a compact operator on the Bloch space. Then a sufficient condition for $F(p, q, s)$ compactness in Theorem 3.4 for a univalent function is

$$
\lim _{|w| \rightarrow 1} \frac{\left.\left.\left|\phi^{\prime}\left(\phi^{-1}(w)\right)\right|^{p-2}\left(1-\mid \phi^{-1}(w)\right)\right|^{2}\right)^{q} g^{s}\left(\phi^{-1}(w), a\right)}{\left(1-|w|^{2}\right)^{q} g^{s}\left(\phi^{-1}(w), a\right)}=0 .
$$

Then

$$
\begin{aligned}
& \lim _{|\phi(z)| \rightarrow 1}\left\{\frac{\left|\phi^{\prime}(z)\right|^{p-2}\left(1-|z|^{2}\right)^{q}}{\left(1-|\phi(z)|^{2}\right)^{q}}\right\} \\
&=\lim _{|\phi(z)| \rightarrow 1}\left\{\frac{\left|\phi^{\prime}(z)\right|\left(1-|z|^{2}\right)}{1-|\phi(z)|^{2}}\right\}^{q}\left(\left|\phi^{\prime}(z)\right|^{\left(\frac{p-2}{q}\right)}\right)=0,
\end{aligned}
$$

or, equivalently,

$$
\lim _{|\phi(z)| \rightarrow 1}\left\{\frac{\left|\phi^{\prime}(z)\right|\left(1-|z|^{2}\right)}{1-|\phi(z)|^{2}}\right\}=0,
$$

by Theorem 3.1 which is a compactness condition for the composition operator on the Bloch space. Hence, by our assumption, $F(p, q, s)$ is a compact operator. For the converse suppose that $C_{\phi}$ is a compact operator on $F(p, q, s)$. By Proposition 3.1, then $C_{\phi}: \mathscr{B} \rightarrow \mathscr{B}$ is a compact operator too.

MacCluer and Shapiro showed that if $C_{\phi}$ is bounded on some weighted Dirichlet space $D_{\alpha}$, then the compactness of $C_{\phi}$ on larger weighted Dirichlet spaces is equivalent to $\phi$ having no angular derivative at each point of $\partial \Delta$ (see [14]). Tjani showed that if $C_{\phi}$ is bounded on some Besov space, then the compactness of $C_{\phi}$ on larger Besov spaces is equivalent to compactness of $C_{\phi}$ on the Bloch space (see [27], Proposition 4.5). We show that if $C_{\phi}$ is bounded 
on some $F(p, q, s)$ space, then the compactness of $C_{\phi}$ on larger $F(p, q, s)$ spaces is equivalent to the compactness of $C_{\phi}$ on the Bloch space.

The theorem above is a special case of the following proposition.

Proposition 3.3. Let $2 \leq r<p^{*}<\infty, 2 \leq p<p^{*}<\infty,-2<$ $q<\infty$ and $0<s<\infty$. Suppose that $C_{\phi}: F\left(r, q_{1}, s\right) \rightarrow F\left(r, q_{1}, s\right)$ for $q_{1}=q /\left(p^{*}-r\right)$ is a bounded operator. Then $C_{\phi}: F(p, q, s) \rightarrow F\left(p^{*}, q, s\right)$ is a compact operator if and only if $C_{\phi}: \mathscr{B} \rightarrow \mathscr{B}$ is a compact operator.

Proof. First, suppose that $C_{\phi}$ is a compact operator on the Bloch space. For any $a \in \Delta$

$$
\begin{aligned}
\left\|C_{\phi} \varphi_{a}\right\|_{F\left(p^{*}, q, s\right)}^{p^{*}}= & \sup _{a \in \Delta} \int_{\Delta}\left|\varphi_{a}^{\prime}(\phi(z))\right|^{p^{*}}\left|\phi^{\prime}(z)\right|^{p^{*}}\left(1-|z|^{2}\right)^{q} g^{s}(z, a) d A(z) \\
= & \sup _{a \in \Delta} \int_{\Delta}\left(\left|\varphi_{a}^{\prime}(\phi(z))\right|^{r}\left|\phi^{\prime}(z)\right|^{r}\left(1-|z|^{2}\right)^{q_{1}} g^{s}(z, a)\right) \\
& \left.\times\left(\left|\varphi_{a}^{\prime}(\phi(z))\right|\left(\left|\phi^{\prime}(z)\right|\left(1-|z|^{2}\right)\right)\right)\right)^{p^{*}-r} d A(z) \\
\leq & \left\|C_{\phi} \varphi_{a}\right\|_{F\left(r, q_{1}, s\right)}^{r}\left\|C_{\phi} \varphi_{a}\right\|_{\mathscr{B}}^{p^{*}-r}
\end{aligned}
$$

for $q_{1}=q /\left(p^{*}-r\right)$ by Theorem 2.2 and since $C_{\phi}: F\left(r, q_{1}, s\right) \rightarrow F\left(r, q_{1}, s\right)$ is bounded, then

$$
\left\|C_{\phi} \varphi_{a}\right\|_{F\left(p^{*}, q, s\right)}^{p^{*}} \leq \text { const. }\left\|C_{\phi} \varphi_{a}\right\|_{\mathscr{B}}^{p^{*}-r} .
$$

Therefore (18) and Theorem 3.3 yield that $\left\|C_{\phi} \varphi_{a}\right\|_{F\left(p^{*}, q, s\right)} \rightarrow 0$ as $|a| \rightarrow$ 1. The converse follows from Proposition 3.2. This finishes the proof of the proposition.

ACKNOWLEDGEMENTS. The authors would like to thank the referee for his valuable remarks and comments.

\section{REFERENCES}

1. Arazy, J., Fisher, S. D., and Peetre, J., Möbius invariant function spaces, J. Reine Angew. Math. 363 (1985), 110-145.

2. Aulaskari, R., and Csordas, G., Besov spaces and the $Q_{q, 0}$ classes, Acta Sci. Math. (Szeged) 60 (1995), 31-48.

3. Aulaskari, R., and Lappan, P., Criteria for an analytic function to be Bloch and a harmonic or meromorphic function to be normal, pp. 136-146 in Complex Analysis and its Applications, Pitman Res. Notes Math. 305, Longman Sci. Tech., Harlow 1994.

4. Aulaskari, R., Xiao, J., and Zhao, R., On subspaces and subsets of BMOA and UBC, Analysis 15 (1995), 101-121. 
5. Aulaskari, R., Stegenga, D. A., and Xiao, J., Some subclasses of BMOA and their characterization in terms of Carleson measures, Rocky Mountain J. Math. 26 (1996), 485-506.

6. Borichev, A., Hedenmalm, H., and Zhu, K. (eds.), Bergman Spaces and Related Topics in Complex Analysis, Contemporary Math. 404, American Mathematical Society, 2006.

7. Bourdon, B. S., Cima, J. A., and Matheson, A. L., Compact composition operators on BMOA, Trans. Amer. Math. Soc. 351 (1999), 2183-2169.

8. Cima, J., and Wogen, W., A Carleson measure theorem for the Bergman space on the ball, J. Operator Theory 7 (1982), 157-165.

9. Conway, J. B., Functions of one Complex Variable, Second Edition, Springer-Verlag, New York 1978.

10. Jiang, L., and He, Y., Composition operators from $\mathscr{B}^{\alpha}$ to $F(p, q, s)$, Acta Math. Sci. Ser. B 23 (2003), 252-260.

11. Li, S., and Wulan, H., Composition operators on $Q_{K}$ spaces, J. Math. Anal. Appl. 327 (2007), 948-958.

12. Lindström, M., Makhmutov, S., and Taskinen, J., The essential norm of a Bloch-to- $Q_{p}$ composition operator, Canad. Math. Bull. 47 (2004), 49-59.

13. Lindström, M., and Palmberg, N., Duality of large family of analytic function spaces, Ann. Acad. Sci. Fenn. Math. 32 (2007), 251-267.

14. MacCluer, B. D., and Shapiro, J. H., Angular derivatives and compact composition operators on the Hardy and Bergman spaces, Canad. J. Math. 38 (1986), 878-906.

15. Madigan, K., and Matheson, A., Compact composition operators on the Bloch space, Trans. Amer. Math. Soc. 347 (1995), 2679-2687.

16. Makhmutov, S., and Tjani, M., Composition operators on some Möbius invariant Banach spaces, Bull. Austral. Math. Soc. 62 (2000), 1-19.

17. Pérez-González, F., and Rättyä, J., Forelli-Rudin estimates, Carleson measures and $F(p, q, s)$ functions, J. Math. Anal. Appl. 315 (2006), 394-414.

18. Rättyä, J., On some complex function spaces and classes, Ann. Acad. Sci. Fenn. Math. Diss. 124 (2001), 1-73.

19. Rättyä, J., The essential norm of a composition operator mapping into the $Q_{s}-$ space, J. Math. Anal. Appl. 333 (2007), 787-797.

20. Rudin, W., Real and Complex Analysis, Third edition, McGraw-Hill, New York, 1987.

21. Shapiro, J. H., Composition Operators and Classical Function Theory, Springer-Verlag, New York, 1993.

22. Smith, W., and Zhao, R., Composition operators mapping into the $Q_{p}$ spaces, Analysis 17 (1997), 239-263.

23. Stroethoff, K., Besov-type characterisations for the Bloch space, Bull. Austral. Math. Soc. 39 (1989), 405-420.

24. Stroethoff, K., The Bloch space and Besov spaces of analytic functions, Bull. Austral. Math. Soc. 54 (1996), 211-219.

25. Tan, H., On subspaces of Bloch space and series with Hadamard gaps, Adv. Math. (China) 27 (1998), 214-223.

26. Tan, H., On subspaces of Bloch space, J. Math. Res. Exposition 20 (2000), 507-510.

27. Tjani, M., Compact composition operators on Besov spaces, Trans. Amer. Math. Soc. 355 (2003), 4683-4698.

28. Wulan, H., Compactness of composition operators from the Bloch space $\mathscr{B}$ to $Q_{K}$ spaces, Acta Math. Sinica 21 (2005), 1415-1424.

29. Zhao, R., On a general family of function spaces, Ann. Acad. Sci. Fenn. Math. Diss. 105 (1996), 1-56.

30. Zhao, R., On $\alpha$-Bloch functions and VMOA, Acta. Math. Sci. 16 (1996), 349-360.

31. Zhu, K., Operator Theory in Function Spaces, Marcel Dekker, New York 1990. 
32. Zhu, K., Analytic Besov spaces, J. Math. Anal. Appl. 157 (1991), 318-336.

\author{
SOHAG UNIVERSITY \\ FACULTY OF SCIENCE \\ DEPARTMENT OF MATHEMATICS \\ 82524 SOHAG \\ EGYPT \\ CURRENT ADDRESS: \\ TAIF UNIVERSIT \\ FACULTY OF SCIENCE \\ MATHEMATICS DEPARTMENT \\ EL-TAIF 5700 \\ EL-HAWIYAH \\ KINGDOM OF SAUDI ARABIA \\ E-mail: ahsayed80@hotmail.com
}

